\title{
Delimitación espacial de unidades territoriales residenciales en la Circunscripción IV - Sección C, Río Gallegos
}

\section{Spatial delimiting of territorial residential units in the Circumscription IV - Section C, Río Gallegos}

\author{
Cristhian González, Alicia P. Cáceres, Cristian A. Ampuero \\ cristhiang_93@hotmail.com,acaceres@uarg.unpa.edu.ar, campuero@uarg.unpa.edu.ar \\ Universidad Nacional de la Patagonia Austral (UNPA), Unidad Académica Río Gallegos \\ (UARG), Instituto de Ciencias del Ambiente, Sustentabilidad y Recursos Naturales \\ (ICASUR) \\ Grupo de Investigación y Extensión Z Terra Cognita, Escuela de Geografía, Ordenamiento \\ Territorial y Geoprocesamiento, Campus Universitario UARG - Av. Gregores y Piloto "Lero" \\ Rivera S/N, Santa Cruz, Río Gallegos
}

Recibido: 21/02/2019. Aceptado: 22/08/2019

\section{RESUMEN}

El siguiente trabajo es producto de una beca de investigación UNPA, la cual tuvo como objetivo reconocer y espacializar las Unidades Territoriales Residenciales (UTR) de la Circunscripción IV, Sección C de la Tercera Franja Residencial (TFR) de la ciudad de Río Gallegos, Provincia de Santa Cruz, Argentina.

Para llevar a cabo esta investigación se recurrió a salidas de campo, recopilación y lectura de bibliografía científica, relevamiento de ordenanzas, cartografía, fotografías aéreas e imágenes satelitales, y se procesó la información recolectada a través de un Sistema de Información Geográfica (SIG).

Para este trabajo, se han identificado las diferentes UTR presentes en el área de estudio, y se relevaron los diferentes usos del suelo.

La hipótesis del trabajo es que el reconocimiento y espacialización de UTR aportarán para la zonificación del uso del suelo que debe realizar el Municipio de la ciudad de Río Gallegos.

Palabras clave: Geografía Urbana; Paisaje Urbano; Rururbano Intraejidal; Territorio; Uso del Suelo residencial.

\footnotetext{
ABSTRACT

The following work is a product of a research fellowship UNPA, which has as purpose the recognition and spatialization of the Territorial Residential Units, of the Circumscription the IV, Section C of the Third Residential Band of the city of Río Gallegos, Province of Santa Cruz, Argentina.

To carry out this research it is resorted to field trips, data collection of scientific bibliography, ordinances survey, cartography, aerial photographs and satellite images, and the information collected was processed through a Geographic Information System.

For this work, the different Territorial Residential Units have been identified in the study area, and the different land uses were surveyed.
} 
The hypothesis of the work is that the recognition and spatialization of Territorial Residential Units will contribute to the land use zoning to be carried out by the city's municipality

Key words: urban geography; urban landscape; rururban intraejidal; Territory; residential ground use.

\section{INTRODUCCIÓN}

El presente trabajo es producto de la actividad realizada por Cristhian González, alumno avanzado de la carrera Licenciatura en Geografía, correspondiente a la Escuela de Geografía, Ordenamiento territorial y Geoinformación de la UNPA - UARG, en el marco de una beca de investigación UNPA dirigida por Alicia P. Cáceres y Codirigida por Cristian Ampuero. El tema de la beca "Delimitación Espacial de Unidades Territoriales Residenciales en la Circunscripción IV - Sección C, Tercera Franja Residencial de Río Gallegos" se enmarca en el Proyecto de Investigación 29A/PDTS 380 "Tercera Franja Residencial de Río Gallegos: Diagnóstico territorial de áreas con ausencia y presencia de cobertura de servicios. Propuesta de localización de Nodos de Servicios".

La hipótesis del trabajo es que el reconocimiento y espacialización de Unidades Territoriales Residenciales (UTR) aportarán para la zonificación del uso del suelo que debe realizar el Municipio de la ciudad de Río Gallegos.

El objetivo del trabajo es reconocer y espacializar UTR de la Circunscripción IV - Sección C como aporte en la zonificación de usos del suelo que debe realizar la Municipalidad.

Estos resultados aportan parcialmente al PI PDTS tanto en el marco teórico, conceptual y metodología.

Se realizaron dos informes, uno de carácter científico, a través de un ICT UNPA, y otro de divulgación, para ser presentado en el municipio como aporte a la zonificación del uso del suelo.

\section{MARCO DE REFERENCIA}

\subsection{MARCO TEÓRICO}

El trabajo se enmarca teóricamente en la Geografía Urbana, la cual es una rama de la Geografía y que según Carter (1983:17) citado en Cáceres (2000:868) "considera los procesos económicos, sociales y políticos, en relación a un solo fenómeno: la ciudad”.

La misma autora, define el concepto de "Ciudad” como: “...un espacio geográfico muy dinámico y complejo; estos rasgos se acentúan a medida que aumenta la diversidad de sus componentes sociales, culturales, económicos, políticos y ambientales. La importante particularidad del territorio santacruceño la constituye la existencia de localidades escasamente pobladas con grandes distancias entre sí, inmersas en un marco natural imponente. Esto hace que su existencia sea singular y que no puedan ser comparadas con otras realidades territoriales del país. Por lo expuesto resulta difícil encontrar una definición satisfactoria y por ende definir si las localidades santacruceñas son ciudades o no. No cabe 
dudas que por las características anteriores consideradas tienen una serie de servicios en lo educativo, asistencial, recreativo que les da el carácter de urbano”. Cáceres (2000:870)

Según Cáceres (2000:868) "Para el desarrollo de la Geografía Urbana de Santa Cruz, se ha optado por el conocimiento de las localidades entendiendo que estas son resultado de un proceso, en el que se articulan dos temas centrales. Por un lado, el Paisaje Urbano y se relación con el medio natural y por el otro, la Estructura Urbana en relación con las actividades que desarrollan, servicios e infraestructura que poseen, distancias entre ellas, etc."

Cáceres et al (2000) para caracterizar el paisaje urbano es necesario recurrir al estudio de la escala intra-urbana, comprendiendo lo referente a las formas urbanas o morfología, la dinámica interna de las ciudades y la ciudad en sí como un área, y deben de tenerse en cuenta tres componentes, a saber: el plano, los usos del suelo y la edificación.

El área de Estudio se corresponde con un tipo de plano ortogonal, el cual se caracteriza por su trazado en formas regulares de manzanas con una tendencia a que las calles se cruzan formando un ángulo recto. Cáceres et al (2000) sostiene que estos tipos de plano "han sido utilizados tanto para fundar ciudades como para el crecimiento de las mismas. Denotan una planificación previa, aunque no significa que esté bien hecha." (Cáceres et al; 2000: 876)

Con respecto a los usos del suelo, Zárate (1984:60), citado en Cáceres et al (2000:879), realiza aportes sobre los usos del suelo, los que caracteriza de la siguiente forma: "Los usos del suelo expresan las diferentes utilizaciones que se hacen del espacio en función de las necesidades y actividades de la población que vive y trabaja en la ciudad".

Entre los diversos usos, ligados a las funciones que se particularizan en la apropiación/transformación del suelo por parte de la población, pueden mencionarse los siguientes:

Administrativo: agrupa organismos ligados a servicios públicos de índole asistencial, educativa y gubernamental.

Comercial: ligado a la concentración de la actividad comercial de diversos rubros y suele estar ubicado en el centro de la ciudad.

Residencial: suele ser el uso de mayor superficie en las ciudades, es el espacio en el cual el hombre reside, se constituye como antagonismo al lugar de trabajo. Cuando no se trabaja, se reside: lo que se conoce como "el hogar".

Industrial: refiere a aquellos establecimientos en los cuales se realiza alguna actividad ligada a alguna etapa del proceso industrial, como por ejemplo, en la provincia de Santa Cruz.

Este trabajo tiene como eje central o foco el uso residencial. Sin embargo, en las situaciones en las que sea necesario se hará mención a los posibles usos del suelo ya sea administrativo, comercial, industrial, etc.

Sobre la tipología arquitectónica, se destaca la patagónica. Cáceres (2000) sostiene que en las mismas "el material de construcción predominante era la chapa; los techos a dos agua - en relación al clima húmedo del invierno - pintados con colores llamativos, no sólo para romper la monotonía del paisaje, sino también, para una mayor visibilidad en la época de nevadas. 
Los ambientes de estancia permanente - cocina y comedor - se diseñan hacia el norte con grandes ventanales para mayor aprovechamiento de la luz y calor del sol. Las aberturas de madera preferentemente y vidrio repartido, también eran pintados con el mismo color del techo." (Cáceres; 2000: 883)

Los mismos autores sostienen que al conjugarse estos tres componentes se tiene como resultado el paisaje urbano. En palabras de Zarate Martin (1991) el mismo representa “... la imagen visual, el lenguaje espacial que expresa estilos de vida, modelos y condiciones de organización socioeconómica que se han sucedido a través del tiempo." (Zárate Martín, 1991:73)

El paisaje urbano puede ir transformándose a lo largo de los años, siendo el uso del suelo el que más varía, y también, la edificación pero en menor medida por todo el tiempo que implica el proceso de construcción. El tipo de plano es el que más perdura en el tiempo, en virtud de la inversión que se realiza para la delimitación de las Circunscripciones, manzanas y parcelas, formando parte de la morfología urbana de un territorio.

En cuanto al plano de la Circunscripción IV - Sección C, a partir de la imagen satelital del área de estudio, puede mencionarse que en dirección al Norte se observa la forma del trazado en forma de cuadrados y rectángulos que hacen a un tipo de plano ortogonal. En dirección al Sur, se observa que la forma del trazado en forma de triángulo.

Zamorano (1992), citado en Norambuena et al (2015:266), define que los barrios son considerados como un “....conjunto que constituye un medio de vida y de actividades, dotado de individualidad. Toda ciudad queda así dividida en sectores homogéneos, espontánea o planificadamente, cuya superficie tiene un destino específico: negocios, industria, administración, residencia."

Según Cáceres (2000) "un barrio residencial es considerado como barrios que ocupan la mayor superficie de las ciudades y está en relación directa a la disociación entre el lugar de habitación y el de trabajo. Las condiciones socioeconómicas, actúan como entradas importantes al sistema espacial, y sus reflejos geográficos se evidencian en la fisonomía de los barrios, en los materiales de construcción, en las modalidades de apropiación de los terrenos en cuanto al tamaño y proximidad de las casas, en la infraestructura vial y de servicios, en la disponibilidad de transporte."

Triviño (2011:24), citado en Aceves (2015:33), realiza una recopilación de conceptos de Barrio en diferentes disciplinas:

- en la Arquitectura, Aronovici (1965) considera que el barrio es el lugar físico o estructura urbana donde se asienta la comunidad vecinal;

- en la Antropología, Ariel Gravano (1991) incorpora la identidad barrial como elemento que define al barrio y que trasciende lo puramente urbano, arquitectónico o espacial, presente en la mayor parte de las definiciones del concepto, el autor lo define como "un lugar común en la ideología de los habitantes de la ciudad, pues tiene una determinada eficacia para referir de un modo sintético a diversos aspectos de la realidad.

- Zamorano (1985), caracteriza a los barrios como secciones de la ciudad que representan cierta identidad o carácter común, generalmente dado por el uso, de esta manera, toda ciudad queda dividida en "sectores homogéneos, espontánea o 
planificadamente, cuya superficie tiene un destino específico, ya sea negocios, industria, administración o residencia;

- dentro de la sociología, Ezequiel Ander-Egg (1995), considera que es una zona o espacio de la ciudad que posee límites físicos y simbólicos (que no coincide necesariamente con la división administrativa de la ciudad)

Según Cáceres (2012:2) “el crecimiento urbano está íntimamente asociado a variables demográficas que, según el ritmo de los desplazamientos (acelerado o lento) y la presencia o no de políticas públicas que orienten el desarrollo territorial, la expansión urbana será descontrolada, fragmentada o por el contrario, armónica, sustentable respectivamente. Ante la demanda habitacional para distintos sectores sociales, la especulación inmobiliaria determina el crecimiento urbano en terrenos periféricos con ausencia de infraestructura y servicios situación que genera la segregación residencial".

Río Gallegos, como toda ciudad patagónica, conforman modelos diferentes a los de otras regiones del país porque han experimentado transformaciones recientes y constantes, además en el territorio provincial de Santa Cruz la extensa superficie y las condiciones físicoclimáticas extremas modifican este tipo particular de crecimiento. En el crecimiento urbano aparecen nuevas formas urbanas en el siglo XXI, son producto de la combinación de distintos factores que responden a realidades locales y no globales; a excepción del aeropuerto internacional, no aparecen las nuevas formas de crecimiento asociadas a los "artefactos de la globalización" que plantea De Mattos en 2001 como producto de la globalización; "shoppings centers, barrios cerrados, edificios inteligentes, centros empresariales, complejos para el esparcimiento, [...] hoteles cinco estrellas, nuevos aeropuertos internacionales, conjuntos empresariales, megaproyectos inmobiliarios, equipamientos de consumo..."). El estudio de crecimiento urbano de Río Gallegos se realiza a partir de la definición de cortes temporales que responden a cambios en los ritmos de crecimiento de la población migrante, atraídos por las posibilidades de trabajo generadas por diversas causales históricas, económicas, políticas, demográficas. Luego, en cada etapa de crecimiento se reconocen elementos de fijación y barreras, en ambos casos naturales y antrópicos, que permitieron e impidieron, respectivamente, el avance urbano como así también la superación de las barreras."

Según Cardoso (2012:32), citado en Cáceres et al (2017:62), "el término rururbano fue propuesto por los franceses G. Bauer y J. M. Roux en 1976, como expresión que identifica a las áreas que rodean a las ciudades antiguas donde, la presencia dominante de viviendas unifamiliares, dispersa y aislada, cohabita con la persistencia de áreas agrícolas y forestales (o naturales)."

Para Sereno et al (2010:43) “...rururbano es la franja externa del periurbano, frontera donde se combinan dos ambientes cuyos pobladores poseen idiosincrasia, forma de vida e intereses particulares y disímiles, si bien sus espacios de vida y referentes espaciales son comunes, pues están signados por la convivencia ... las técnicas y herramientas de trabajo propios del ambiente rural se combinan con la infraestructura, el equipamiento y los servicios urbanos para forjar un entramado distintivo y frágil. De este modo se genera un ámbito propio de acción que posee un ritmo singular, caracterizado por la interacción y competencia de múltiples intereses, usos del suelo y prácticas propias de un espacio de transición o "frontera", en términos de Barros y Zusman (2001:27)”.

El Estado municipal tiene dos formas de dividir y denominar los sectores del ejido que no son coincidentes ni articuladas entre sí. Por un lado, "la designación catastral" que abarca todo el 
ejido municipal y comprende los aspectos fiscales y de registro dominial de la parcela y establece unidades que, yendo de mayor a menor, son: la Circunscripción ( 7 denominadas en números romanos), las secciones (denominadas en letras mayúsculas y varían la cantidad según la Circunscripción), las manzanas y las parcelas.

Para el plan de trabajo de la beca de investigación, se ha seleccionado como área de estudio la Circunscripción IV, Sección C, que se extiende al S-SO de la ciudad.

Si bien los usos permitidos son el residencial, industrial mixto y rural, por los bajos valores inmobiliarios de la subdivisión de chacras se han comprado más de dos lotes lindantes para usos como el comercial mayorista, depósitos, talleres, lavaderos de transporte pesado, empresas de transportes pesados, con respecto a los usos deportivos en ambientes cubiertos y al aire libre se encuentran en proceso incipiente pero cabe aclarar que no son construidos por el Estado. Como resultado, se observa una desorganizada ocupación por la presencia de diferentes usos del suelo y en algunos casos, por la incompatibilidad de usos del suelo. A los efectos de una eficiente gestión del territorio, es necesaria la redacción de una ordenanza de usos del suelo y zonificación. El reconocimiento de UTR aportará a la generación de una herramienta básica de zonificación del área.

Un Sistema de Información Geográfica (SIG) es un sistema de hardware, software y procedimientos elaborados para facilitar la obtención, gestión, manipulación, análisis, modelado, representación y salida de datos espacialmente referenciados, para resolver problemas complejos de planificación y gestión (NCGIA; 1990).

\subsection{MARCO CONCEPTUAL}

La Real Academia Española (RAE) define unidad como: "Propiedad de todo ser, en virtud de la cual no puede dividirse sin que su esencia se destruya o se altere".

Tomando la definición del Diccionario de Geografía Urbana, Urbanismo y Ordenación del Territorio, Unidad Territorial es la "División de un territorio en partes al efecto de su estudio, planificación y ordenación. Puede hacerse con propósito meramente analítico o como paso previo a la delimitación de zonas sobre las que recae un régimen diferenciado de protección o gestión. La distinción de unidades territoriales se realiza siguiendo criterios diversos; a partir de rasgos constitutivos diferenciadores o integrando áreas que se complementen, respetando las delimitaciones administrativas preexistentes o proponiendo nuevas demarcaciones. En el primer caso se adjetivan según el punto de vista principal de estudio: unidad de paisaje, unidad hidrológica; en el segundo según los objetivos del plan: unidades de diagnósticos, unidades de actuación, aunque reconociendo siempre en ellas los caracteres propios de cada parte del territorio." (Zoido. 2000:307)

Para Ocampo Marín (2005:3), citado en Spataro (2008), el territorio "es un constructo social, una valoración, una fabricación, un producto, un espacio tatuado por la historia y la cultura, que se construye a través de prácticas, estrategias, percepciones y la manera que tienen de leer los miembros del grupo que los constituye".

Según Laurelli (2004:434) el territorio "Es la malla de gestión del espacio que tiene como principio un estatuto inferior a las circunscripciones normales porque la apropiación no parece completamente realizada. Implica una apropiación del espacio e identificación colectiva e individual, con formas de exclusión, agregación, segregación." 
"El territorio da testimonio de una apropiación a la vez económica, ideológica y política (social por lo tanto) del espacio, por grupos que se dan una representación particular de ellos mismos, de su historia y de su singularidad" (Di Méo, 2000). El concepto de territorio reúne las nociones del espacio social y de espacio vivido. Describe, apoyándose sobre datos (espaciales) de la geografía, la inserción de cada sujeto en uno o más grupos sociales de referencia, en su dimensión política traduce un modo de recorte y de control del espacio que garantiza la especificidad y la permanencia, la reproducción de los grupos humanos que lo ocupan y ordenado por las sociedades que lo han construido sucesivamente, en tercer lugar constituye un campo simbólico".

Reconocimiento territorial, por el Diccionario de Geografía Urbana, Urbanismo y Ordenación del Territorio, lo definen como "conjunto de estudios referidos a un ámbito o un territorio definido, generalmente previos a investigaciones explicativas, a la realización de un plan de ordenación o a la definición de políticas territoriales explícitas. De modo aún más genérico, las expresiones reconocimiento de territorios o reconocimiento de tierras (land reasearch, land survey) han sido utilizadas en metodologías de conocimiento del paisaje y en la descripción de espacios útiles con posterioridad a otros propósitos e interpretaciones. En ellas, la observación directa o mediante fotointerpretación del terreno de algunas de sus variables más básicas (topografía, vegetación, sueldos) es seguida de análisis que aprecian las coincidencias espaciales y proponen el establecimiento de unidades y tipologías formales, finalizando con estudios de integración y definición de la estructura de cada tipo o unidad territorial". (Zoido; 2000:359)

A modo de síntesis, se define que una Unidad Territorial Residencial es un espacio que demuestra homogeneidad y cohesión territorial particularmente por constituirse como un área en donde predomina el uso del suelo residencial y que presenta características geográficas, históricas, arquitectónicas y sociales que le otorgan uniformidad.

\subsection{MARCO HISTÓRICO - GEOGRÁFICO}

Río Gallegos es la capital de Santa Cruz. Es una ciudad intermedia que presenta las características de las ciudades de la Patagonia Austral: ocupación reciente y crecimiento demográfico con un importante componente migratorio. El sitio de Río Gallegos es de $51^{\circ} 38^{\prime}$ Latitud Sur y $69^{\circ} 17^{\prime}$ Longitud Oeste, siendo la ciudad más austral de la Patagonia Continental Argentina. (fig. 1)

Según Cáceres et al (2016:52) "Es la más poblada y [...] depende de una metrópoli extrarregional, la Ciudad Autónoma de Buenos Aires. Además se superponen a lo largo de 35 $\mathrm{km}$, las Rutas Nacionales (RN) $\mathrm{N}^{\circ} 3(\mathrm{~km} 2607)$ y $\mathrm{N}^{\circ} 40(\mathrm{~km} \mathrm{135),} \mathrm{que} \mathrm{definen,} \mathrm{para} \mathrm{Río}$ Gallegos, la situación de encrucijada. (Cáceres, 1996; 2013). [...]”. 
Fig. 1: Localización de Río Gallegos

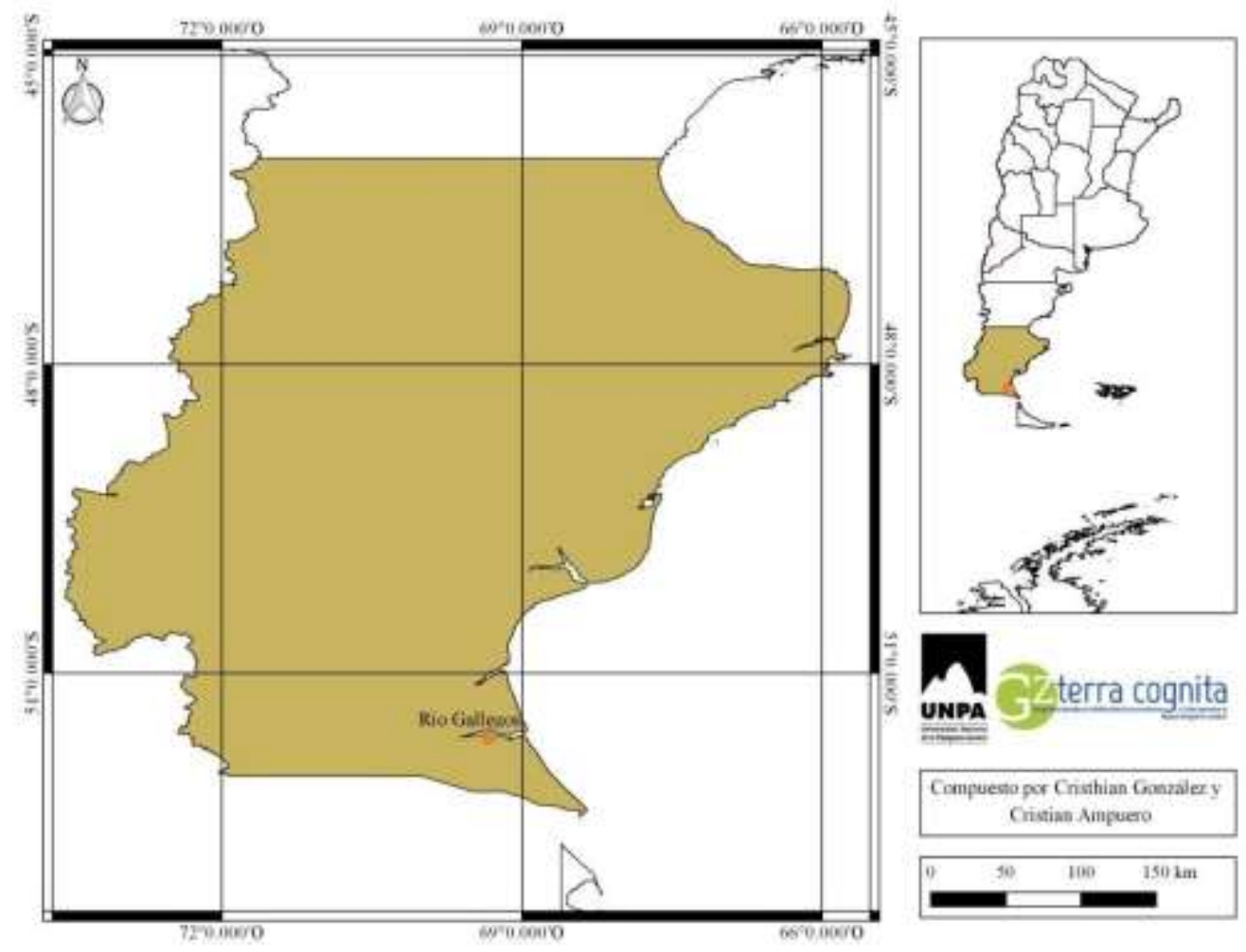

Fuente: Elaboración propia

Según los mismos autores, la $\mathrm{RN} \mathrm{N}^{\circ} 3$ es el eje estructurador en sentido N-S del E santacruceño y de la Patagonia Austral, que, dentro de Santa Cruz es la principal vía de comunicación terrestre con Tierra del Fuego y la XII ${ }^{\circ}$ Región de Chile con el resto de sus respectivos países. Por otro lado, la $\mathrm{RN} \mathrm{N}^{\circ} 40$ nace en el extremo SE de la Provincia de Santa Cruz, en Cabo Vírgenes. Desde Río Gallegos se dirige hacia el W hasta Río Turbio y 28 de Noviembre- que comunican con las localidades chilenas de Puerto Natales y Punta Arenas.

Hacia el N, comunica a través de rutas provinciales (RP) con las localidades turísticas de El Calafate y El Chaltén. Luego con Gobernador Gregores y Perito Moreno. Cuenta con un Aeropuerto Internacional cuyas frecuencias y destinos varían, principalmente, son a Buenos Aires, Río Grande, Ushuaia y Comodoro Rivadavia. Constituye el último punto de apoyo continental de las comunicaciones con Antártida y las Islas Malvinas. Es una ciudad no primada en proceso de metropolización, con equipamiento en servicios e infraestructura suficiente que la definen como ciudad intermedia.

Según el último Censo, registró 95.796 habitantes (INDEC, 2010), que equivale al 35\% de la población, que la ubica en el rango 1 del sistema urbano provincial de 15 localidades. Su función política administrativa, por ser capital de provincia, y localización en área de frontera, está en un permanente aumento de complejidad de sus servicios." Según la RAE "acción y efecto de servir", y servir es estar al servicio de alguien.

Para Cáceres (2013:2) la ciudad de Río Gallegos “...está emplazada sobre la margen sur del estuario del río Gallegos, sobre terrazas fluviales y algunos paleocauces, a una altura media por debajo de los 20 m.s.n.m. Se encuentra ubicada desde el punto de vista fisiográfico en el denominado Patagonia Austral Extraandina (dentro del ecosistema Estepa Magallánica), con 
ausencia absoluta de árboles, propio del tipo de clima de esta zona, frío semiárido de meseta con precipitaciones que no superan los $300 \mathrm{~mm}$ anuales. La latitud es un condicionante de la vida urbana puesto que corresponde a la zona de libre circulación atmosférica y oceánica; que explica la presencia de vientos fuertes del Oeste, la falta de verano térmico y los días largos del verano y las noches largas del invierno. Por lo tanto, el emplazamiento se caracteriza por la aridez, vientos fuertes y mesetas de suave relieve que generan un paisaje natural monótono y hostil”.

\section{RESULTADOS, ANÁLISIS Y DISCUSIÓN}

\subsection{METODOLOGÍA}

En un primer lugar se llevó a cabo un relevamiento de ordenanzas, cartografía, fotografías aéreas e imágenes satelitales del área de estudio a través de los programas Google Earth y Google Street View.

El Archivo Histórico Municipal, permitió el acceso a la cartografía perteneciente a la década del 60', con la cual se pudo dar cuenta de las chacras históricas de la Circunscripción que se encuentran subdivididas, mientras que el Municipio permitió el acceso a las ordenanzas municipales para conocer el marco legal de las Circunscripciones.

Luego, se realizó una recopilación y lectura de bibliografía específica en bibliotecas reales como virtuales para profundizar sobre las UTR y áreas rururbanas y periurbanas.

Se realizó una primera salida de campo, para cumplir con los objetivos de reconocimiento del área de estudio mediante la observación directa.

Luego en trabajo de gabinete, se comenzó con la digitalización de las UTR en un Sistema de Información Geográfica (SIG). El software utilizado para llevar a cabo esta actividad fue el QGIS en su versión 2.18 "Las Palmas".

Una vez delimitadas todas las UTR, se llevó a cabo una recopilación de los diferentes usos del suelo, a través del uso de imágenes satelitales, obtenidas con el programa Google Earth, sobre una planilla previamente diseñada en gabinete.

Se llevó a cabo una segunda salida de campo, para corroborar y completar con la información necesaria. A su vez se obtuvieron las coordenadas geográficas a través del GPS del celular y toma de fotografías.

Se le asignó una letra del alfabeto a cada unidad territorial. En el caso que se encontraron subdivisiones, se le asignó una letra y un número.

A partir de esta información se llevó a cabo la elaboración de la cartografía.

Por último, se pasó a la elaboración del Informe Científico Técnico. 


\subsection{RESULTADOS}

A modo de síntesis, la posición geográfica de la Circunscripción IV, Sección C es (fig. 2):

Con respecto al sitio es:

- Norte, 51 $1^{\circ} 37^{\prime} 32.15^{\prime \prime}$ Latitud Sur, 69¹5'8.05" Longitud Oeste.

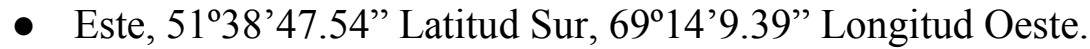

- Sur, 51 $41^{\prime} 42.17^{\prime \prime}$ Latitud Sur, 69¹6'31.73" Longitud Oeste.

- Oeste, 51³9'5.22” Latitud Sur, 69¹6’26.10” Longitud Oeste.

Fig. 2: Ubicación del área de estudio
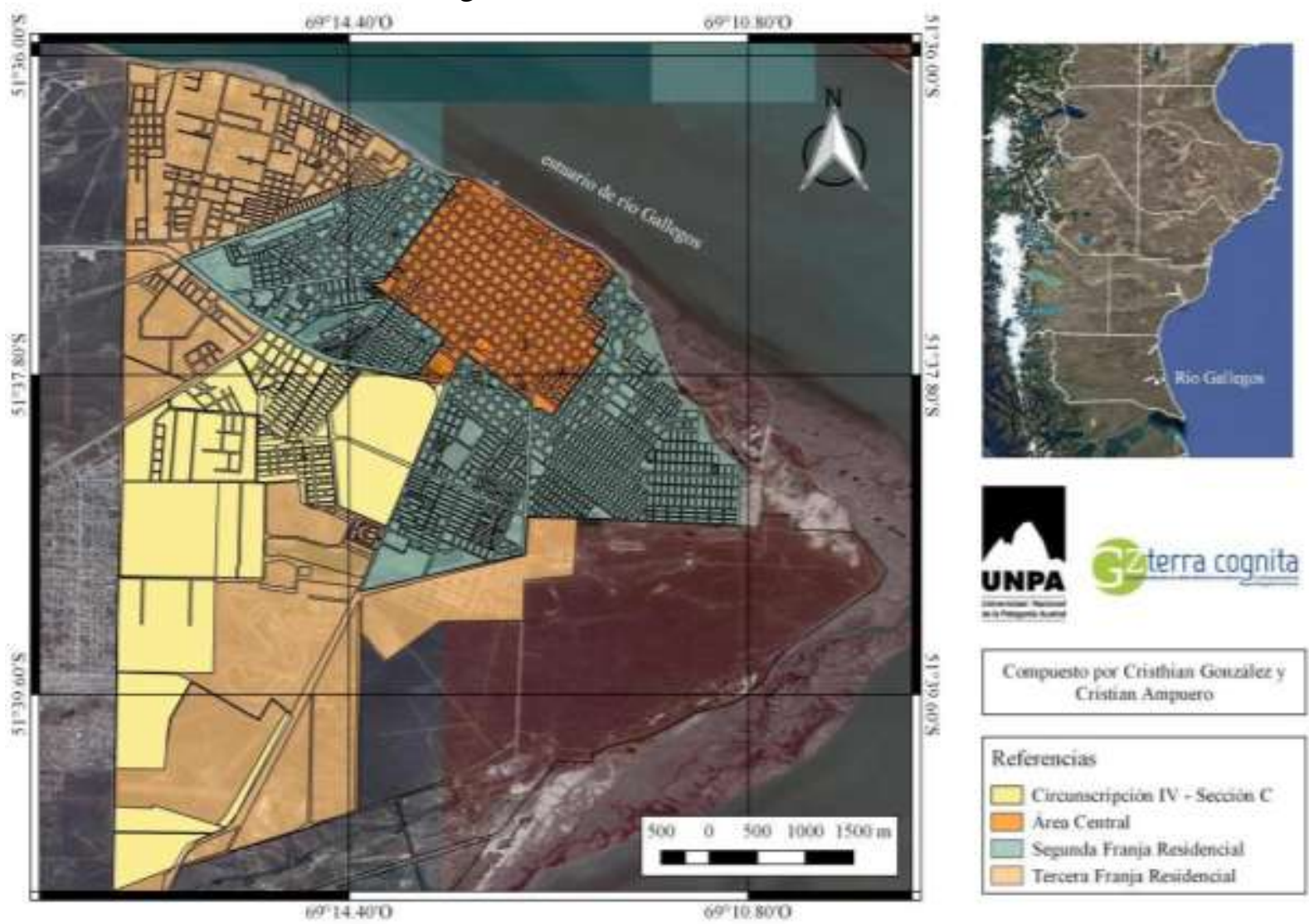

Cempuesto por Cristhian Gourallez y Cristian Ampoero

Referencias
$\square$ Circunscripción IV-Seccice C
Ares Central
Sepunda Franja Residencial
Tercera Franja Residencial

Fuente: Elaboración propia, modificado de Cáceres et al (2013), tomando como base imagen satelital de Google Earth

En cuanto al Emplazamiento, al igual que el de la ciudad de Río Gallegos, es atravesado desde el Este al Oeste por un paleocauce, de una profundidad aproximada por $10 \mathrm{~m}$, continuidad de la laguna Ortiz (fig. 3). 
Fig. 3: Perfil de elevación, Paleocauce de laguna Ortiz

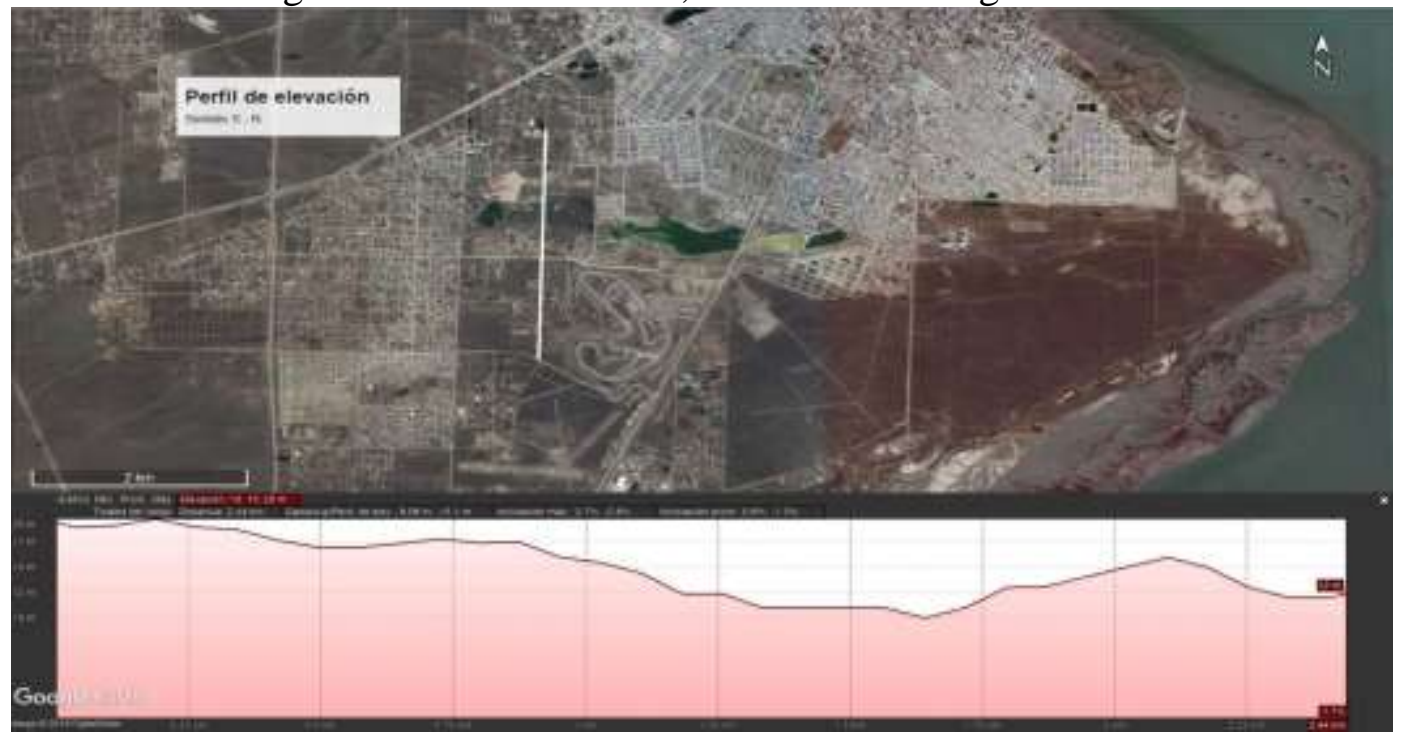

Fuente: Google Earth

La Situación es extrema con respecto a la ciudad, se comunica con el resto de Río Gallegos a través de la Av. Asturias, la Autovía 17 de Octubre y la Av. San Martín.

En cuanto a la Estructura Urbana Interna, el área de estudio, que comprende una superficie aproximada de 10,43 $\mathrm{km}^{2}$, pertenece a la TFR el sector sur comprendido entre Autovía 17 de Octubre, Av. San Martín, Calle 1 y Av. Asturias.

En el sector sur se conformó a partir chacras improductivas que han sido loteadas por sus propietarios y vendidas para el uso residencial con servicios básicos de energía, gas en red, agua pero con ausencia de Nodos de Servicios que, según Cáceres y Ampuero (2008), son entendidos como un conjunto de unas pocas manzanas contiguas localizadas en puntos estratégicos y accesibles en el área al que pertenecen, y que "...comprenden equipamiento en servicios educativos, de salud, recreativos, comerciales, financieros, seguridad, asistencia social y de sanidad, entre otros que mejoren la calidad de los vecinos" (Cáceres y Ampuero, 2008:180).

Se reconocieron y espacializaron seis (6) UTR en la Circunscripción IV, Sección C. (fig. 4), y se establecieron los límites que figuran en la tabla 1. 
Fig. 4: Distribución de las UTR en la Circunscripción IV, Sección C

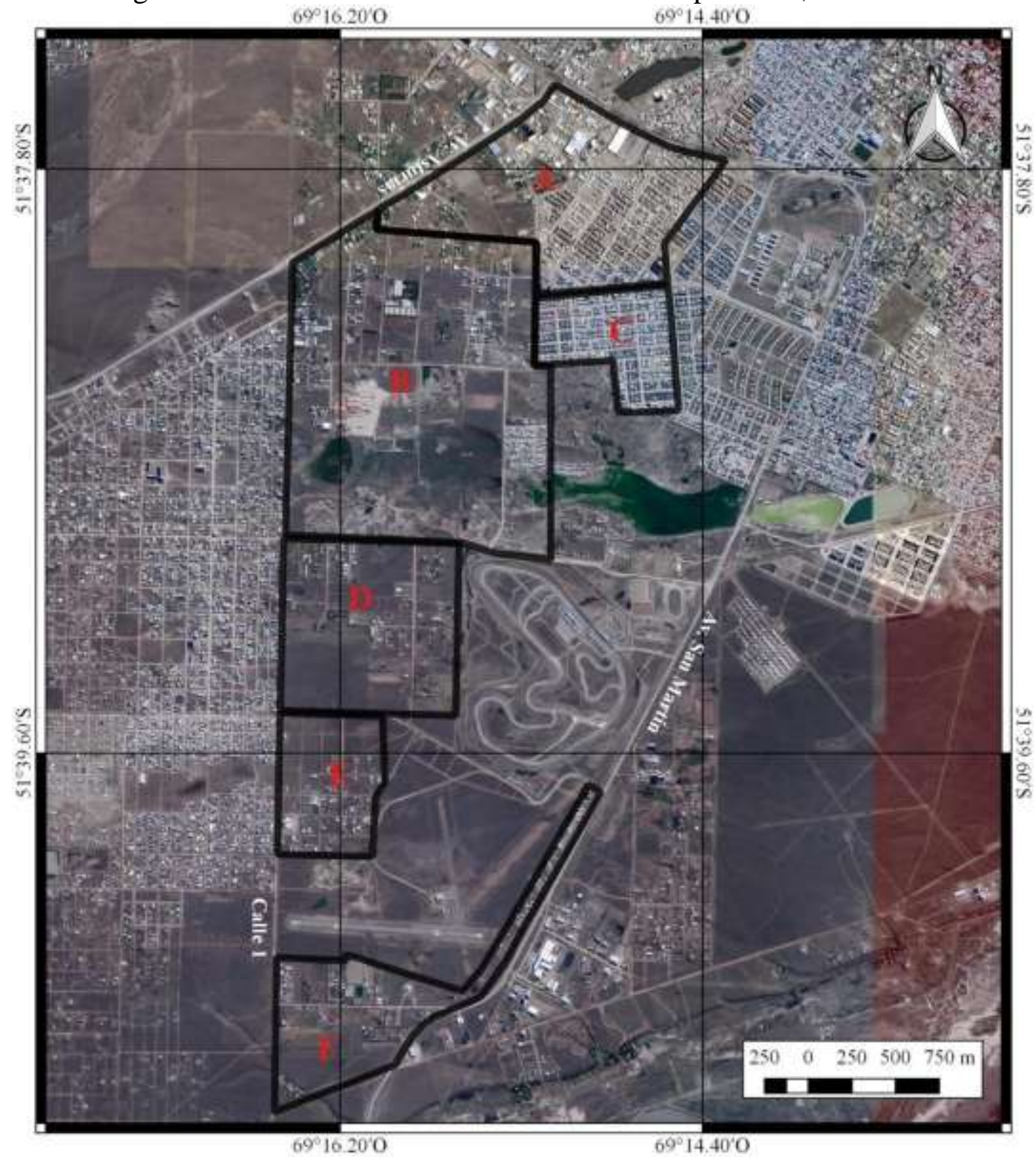

\section{Referencias}

Unidades Territoriales Residenciales

Compuesto por Cristhian Gonzalez y Cristian Ampuero

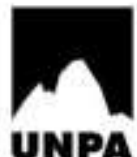

itiventine

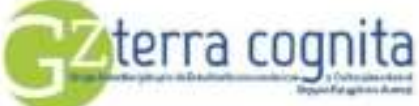

Fuente: Elaboración propia tomando como base imagen satelital de Google Earth 
Tabla 1: Límites de las UTR

\begin{tabular}{|c|c|c|c|c|}
\hline \multirow{2}{*}{$\begin{array}{c}\text { Unidades } \\
\text { Territoriales } \\
\text { Residenciales }\end{array}$} & \multicolumn{4}{|c|}{ Límites } \\
\hline & Norte & Sur & Este & Oeste \\
\hline \multirow{3}{*}{$\mathbf{A}$} & Av. Asturias & $\begin{array}{c}\text { Emilio Roldán } \\
\text { Molina }\end{array}$ & $\begin{array}{c}\text { Mahatma } \\
\text { Gandhi }\end{array}$ & Miguel Ángel \\
\hline & $\begin{array}{l}\text { Av. Asturias - } \\
\text { Paseo de los } \\
\text { Arrieros }\end{array}$ & Félix Riquez & Juan Esteban & $\begin{array}{l}\text { Dr. Boris Gos - } \\
\text { Mahatma Gandhi }\end{array}$ \\
\hline & $\begin{array}{l}\text { Paseo de los } \\
\text { Arrieros }\end{array}$ & Félix Riquez & $\begin{array}{l}\text { Luis Gotti - } \\
\text { Jorge Luis } \\
\text { Lorenzo }\end{array}$ & Juan Esteban \\
\hline B & Félix Riquez & $\begin{array}{c}\text { Maestra Sara } \\
\text { Mignone de } \\
\text { Oliveira - Calle } \\
\text { sin nombre - } \\
\text { Pionero Joaquín } \\
\text { González }\end{array}$ & $\begin{array}{l}\text { Jorge Luis } \\
\text { Lorenzo }\end{array}$ & Dr. Boris Gos \\
\hline
\end{tabular}

Av. Asturias -

C Miguel Ángel -

Emilio Roldán

Molina

Palle $22 \quad \begin{gathered}\text { Pionero Joaquín } \\ \text { González - } \\ \text { Sandalio Vera }\end{gathered}$

Dr. Boris Gos -

González -

Calle 1

\begin{tabular}{ccccc}
\hline D & Calle 22 & Calle 38 & Calle S/N & Calle 1 \\
\hline E & Calle 38 & Dunncanmann & Calle S/N & Calle 1 \\
\hline F & Dunncanmann & Calle S/N & $\begin{array}{c}\text { Av. José de San } \\
\text { Martín }\end{array}$ & Calle 1 \\
\hline
\end{tabular}

Fuente: Elaboración propia, con datos relevados por el autor en el mes de junio del 2018

Una vez reconocidas las UTR, se identificaron y analizaron los elementos que constituyen el paisaje urbano del área de estudio: uso del suelo (tabla 2), proceso de consolidación (tabla 2), entramado (tabla 3) y tipología de vivienda (tabla 4). 
Tabla 2: Usos del Suelo

\begin{tabular}{ccccc}
\hline \multirow{2}{*}{ UTR } & \multicolumn{4}{c}{ Usos del Suelo } \\
\cline { 2 - 4 } & Residencial & Comercial & Administrativo & Industrial \\
\hline A & $\mathrm{X}$ & $\mathrm{X}$ & $\mathrm{X}$ & \\
B & $\mathrm{X}$ & & & \\
C & $\mathrm{X}$ & $\mathrm{X}$ & & \\
D & $\mathrm{X}$ & & & \\
E & $\mathrm{X}$ & & & \\
F & $\mathrm{X}$ & & & \\
\hline
\end{tabular}

Fuente: Elaboración propia con datos relevados por los autores

Tabla 3: Proceso de ocupación

\begin{tabular}{ccc}
\hline Unidad & \multicolumn{2}{c}{ Proceso de ocupación } \\
\cline { 2 - 3 } Territorial & Consolidado & Incipiente \\
\hline A & $\mathrm{X}$ & \\
B & $\mathrm{X}$ & \\
C & $\mathrm{X}$ & \\
D & & $\mathrm{X}$ \\
E & & $\mathrm{X}$ \\
F & & $\mathrm{X}$ \\
\hline
\end{tabular}

Fuente: Elaboración propia con datos relevados por los autores

Tabla 4: Entramado

\begin{tabular}{|c|c|c|}
\hline \multirow{2}{*}{$\begin{array}{c}\text { Unidad } \\
\text { Territorial } \\
\text { Residencial } \\
\end{array}$} & \multicolumn{2}{|c|}{ Entramado } \\
\hline & Abierto & Cerrado \\
\hline $\mathbf{A}$ & & $\bar{X}$ \\
\hline B & $\mathrm{X}$ & \\
\hline $\mathbf{C}$ & & $\mathrm{X}$ \\
\hline D & $X$ & \\
\hline $\mathbf{E}$ & $X$ & \\
\hline $\mathbf{F}$ & $X$ & \\
\hline
\end{tabular}

Fuente: Elaboración propia con datos relevados por los autores

Tabla 5: Tipo de vivienda / Tipología Arquitectónica

\begin{tabular}{|c|c|c|c|c|}
\hline \multirow{2}{*}{$\begin{array}{c}\text { Unidad } \\
\text { Territorial } \\
\text { Residencial }\end{array}$} & \multicolumn{2}{|c|}{ Tipo de vivienda } & \multicolumn{2}{|c|}{ Tipología Patagónica } \\
\hline & Unifamiliar & Multifamiliar & $\mathbf{S i}$ & No \\
\hline $\mathbf{A}$ & $\mathrm{X}$ & & $\mathrm{X}$ & \\
\hline B & $X$ & & $X$ & \\
\hline $\mathbf{C}$ & $\mathrm{X}$ & & $\mathrm{X}$ & \\
\hline D & $\mathrm{X}$ & & $\mathrm{X}$ & \\
\hline $\mathbf{E}$ & $\mathrm{X}$ & & $X$ & \\
\hline $\mathbf{F}$ & $X$ & & $X$ & \\
\hline
\end{tabular}




\subsection{DISCUSIÓN}

Se correlacionaron las variables presentadas en las tablas de análisis (uso del suelo, proceso de ocupación, entramado y tipo de vivienda). A continuación se analizan y representan (fig. 5) las UTR identificadas.

Fig. 5: Proceso de ocupación, entramado y tipología arquitectónica en las UTR

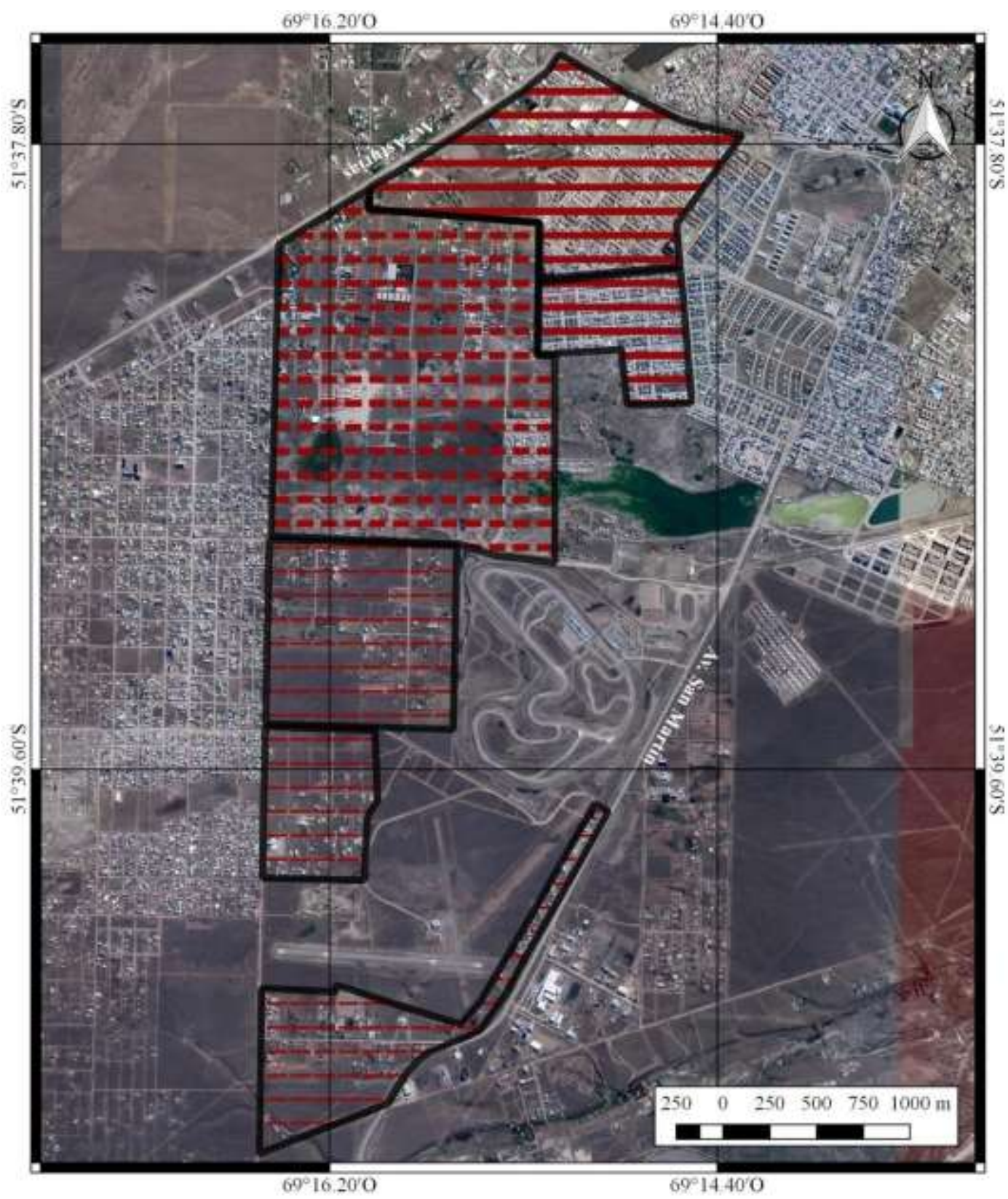

Leyenda

Proceso de ocupación, entramado y tipologia arquitectónica en las UTR

- Proceso de ocupación consolidado, entramado cerrado con tipologia patagónica

- Proceso de ocupación consolidado, entramado abierto con tipologa patagónica

- Proceso de ocupación incipiente, entramado abierto con tipologia patagòica

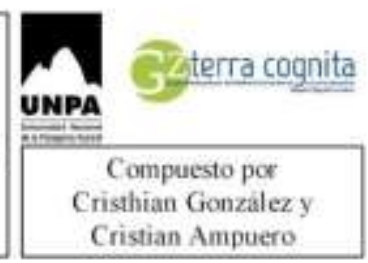

Fuente: Elaboración propia tomando como base imagen satelital de Google Earth 


\section{UTR A:}

El uso de suelo predominante es el Residencial. El proceso de ocupación es consolidado. Presenta un entramado de tipo cerrado y las viviendas poseen una tipología patagónica. Con respecto al tipo de vivienda es de tipo unifamiliar.

En el uso comercial, encontramos el Hipermercado "Carrefour", mayorista "Gualdesi Hnos.", centro de distribución "La Anónima" y la heladería "Grido".

En el uso Administrativo, encontramos un establecimiento donde funciona la Escuela $\mathrm{N}^{\circ} 62$ "René Gerónimo Favaloro" y Escuela No 90 "Hugo Giménez Agüero". Otro establecimiento educativo es el Colegio Secundario No 36 "Macá Tobiano".

Se localiza la terminal de ómnibus de Río Gallegos "Manuel Álvarez” y el Instituto Nacional de Tecnología Agropecuaria (INTA).

También se encontró uso del suelo recreativo: una cancha de césped sintético "La gambeta", el complejo del Sindicato de Empleados de Comercio y diferentes confiterías bailables nocturnas.

\section{UTR B:}

El uso del suelo predomina el uso residencial con un proceso de ocupación consolidado. Presenta un entramado cerrado, con una tipología de tipo patagónica. El tipo de vivienda que prevalece es el unifamiliar.

Dentro del uso del suelo recreativo, encontramos el Polideportivo de la MPCSS Salón Recreativo y un salón para eventos llamado "La Carbonería".

\section{UTR C:}

Con respecto al uso del suelo impera el tipo residencial, con una ocupación que se encuentra en proceso de consolidación acelerado. El entramado es abierto y la tipología de vivienda es la patagónica. El tipo de vivienda es unifamiliar.

Dentro del uso comercial, encontramos el Autoservicio "Cerca", Ferretería "Nueva Santa Cruz" y Veterinaria y peluquería canina "Huellitas".

\section{UTR D:}

El uso del suelo es de tipo residencial, con un proceso de ocupación incipiente. Posee en su mayoría un entramado abierto, con una tipología de vivienda de tipo patagónica y predominio de viviendas unifamiliares.

Dentro del uso educativo, se encuentra "La granjita educativa", un proyecto que tuvo como iniciativa de organizar visitas a su predio, con el objetivo de que los niños de nivel inicial, se interioricen y conozcan sobre los animales, enfocado en el cuidado de la naturaleza.

En el uso recreativo encontramos un salón de eventos del Sindicato Argentino de Televisión, Servicios Audiovisuales, Interactivos y de Datos (SATSAID) y el autódromo "José Muñiz", 
que es un circuito de carreras para competiciones de deporte motor, en el cual se llevan a cabo los campeonatos de Turismo Carretera y TC Pista y Turismo nacional.

\section{UTR E:}

El tipo de uso de suelo predominante es el residencial, con un proceso de ocupación incipiente. Presenta un entramado abierto con una tipología de vivienda patagónica y de tipo unifamiliar.

Dentro del uso religioso, encontramos la iglesia cristiana auditorio "Bet El".

Se localiza el Aeroclub Río Gallegos "Dr. Ernesto Pueyrredón”, el mismo posee alrededor de 100 ha. y una pista de Asfalto, con una orientación 26/08 y un largo de 1100 m. y 23 m. de ancho.

Se llevan a cabo diferentes actividades como una escuela de vuelo, bautismos de vuelos, vuelos panorámicos, traslados, vuelos de observación, entre otras.

Es el primer aeropuerto de Río Gallegos y lleva recibiendo vuelos desde la década de 1.920, entre ellos el piloto Antoine de Saint-Exupéry, autor de "El Principito".

Se destaca la presencia de una cantera no remediada. Se observaron mucha cantidad de perros, pero no en la calle, sino en el patio o frente de la vivienda.

En este sector, se distinguen como quintas y no como chacras. Se observan casas pensadas para quedarse, con estilos propios, y han pagado a personas para que diseñen y construyan la vivienda, eso quiere decir que las personas no tienen pensado armar una vivienda para estar un tiempo y dejar la ciudad, sino que con finalidad de quedarse a vivir en Río Gallegos.

En el barrio "Los Sauces", se ubica sobre un paleocauce suave. Podemos observar que la gran mayoría de las casas tienen un cartel en sus viviendas que dice: "Vecinos en alerta. Barrio Los Sauces". Esto da a conocer que ya están trabajando como vecinos de un barrio, con identidad propia.

Además, cuenta con la presencia de un taller de carpintería metálica e instalaciones eléctricas.

\section{UNIDAD F:}

El uso de suelo predominante es el residencial con un proceso de ocupación incipiente. Posee en su mayoría un entramado de tipo abierto, con una tipología patagónica y viviendas unifamiliares.

Dentro del uso residencial, se encuentran viviendas que son sorteadas en las rifas de "Héctor Raúl Barragán”.

En el uso recreativo encontramos una cancha de futbol y en el comercial el Motel "Eros".

Dentro de la Circunscripción IV, Sección C, un 25,22\% no corresponden al uso residencial, destacando lugares ocupados por el autódromo "José Muñiz", el aeródromo "Dr. Ernesto Pueyrredón" y el sector occidental de la laguna Ortiz. 
A modo de síntesis y luego del análisis realizado, se determinó que la confluencia de la Avenida Asturias con la Autovía "17 de Octubre" actúa como ejes de fijación para el proceso de ocupación consolidado y continúo para el uso residencial, acompañado de un entramado cerrado y con elementos de la tipología patagónica, como el techo a dos aguas.

A medida que nos dirigimos hacia el sur por la Av. Asturias y siguiendo por la Calle 1, ésta consolidación va desapareciendo y es sustituida por el entramado de tipo abierto, manteniendo la tipología patagónica, a lo largo de toda la Circunscripción.

Se observa una tendencia a la no homogeneidad, ya que se encuentra solo en el sector más cercano a la Av. Asturias y Autovía "17 de Octubre".

\section{CONCLUSIÓN}

Respecto a la hipótesis planteada inicialmente, la misma se confirma. Se reconocieron y espacializaron las diferentes Unidades Territoriales Residenciales, generando cartografía base para futuros trabajos de zonificación por parte de los gestores del territorio.

La metodología adoptada ha sido la correcta ya que ha permitido que los objetivos se cumplan de manera efectiva, integrando la información relevada en un SIG.

Esta beca de investigación realiza un gran aporte para la formación del becario como futuro Lic. En Geografía, poniendo en práctica la redacción científica y utilizar diferentes herramientas para generar cartografía.

Por último, se destaca que los resultados aportan parcialmente al PI 29A/380 PDTS, sobre marco teórico, conceptual y metodología.

\section{AGRADECIMIENTOS}

A la Lic. Alicia P. Cáceres y al Lic. Cristian A. Ampuero por el ofrecimiento a participar en ésta experiencia, su acompañamiento a lo largo de la beca, su buena predisposición y la transmisión de conocimientos y seguridad.

A Merlina Saldivia, alumna avanzada de la carrera Lic. en Trabajo Social, por su compañerismo y su buena voluntad para trabajar en equipo.

Al Proyecto de Investigación 29A/380 PDTS “Tercera Franja Residencial de Río Gallegos: Diagnóstico territorial de áreas con ausencia y presencia de cobertura de servicios. Propuesta de localización de Nodos de Servicios" que financió las salidas de campo e impresión del informe final.

A la Universidad Nacional de la Patagonia Austral por financiar la beca de investigación en el marco de la cual se desarrolló este trabajo de investigación.

Al Archivo Histórico Municipal de Río Gallegos por permitir la revisión de los expedientes consultados.

A mis padres, hermanos y amigos, por el apoyo recibido. 


\section{REFERENCIAS}

ACEVES, K. 2015. "Identificación Geográfica de barrios residenciales surgidos a partir de la actividad hidrocarburífera en Río Gallegos durante el Siglo XX.” Informe Científico Técnico UNPA, Vol. 7, No. 2. Río Gallegos. https://doi.org/10.22305/ictunpa.v7i2.126

CÁCERES, A. P. 2000. "Estructura y paisajes urbanos de Santa Cruz. El Gran Libro de Santa Cruz." Alfa Centro Literario y Editorial Oriente. España.

CÁCERES, A. 2013. "Crecimiento urbano de la ciudad de Río Gallegos, Capital de la Provincia De Santa Cruz, Patagonia austral argentina (1885-2010)”. 2013. Revista Electrónica GEOUSAL. Revista Científica de Geografía No 14.

https://www.usal.edu.ar/archivos/geousal/docs/crecimiento_urbano_rio_gallegos_18852010.pdf https://doi.org/10.14198/inturi2015.10.09

CÁCERES, A. P.; Ampuero, C. 2008. "Análisis de la distribución espacial de nodos de servicios en la segunda franja residencial de una ciudad intermedia patagónica como aporte al diagnóstico territorial.” En: Boletín Geográfico No 31 . Edición Especial No 3. VII Jornadas Patagónicas de Geografía. Universidad Nacional del Comahue. Neuquén. https://doi.org/10.21840/siic/158081

CÁCERES, A. P.; García, A.N. 2002. "Transformaciones en el paisaje urbano de Río Gallegos, Patagonia Argentina." En: Congreso Nacional de Geografía $63^{\circ}$ Semana de Geografía. Contribuciones Científicas, GÆA. Buenos Aires. https://doi.org/10.19137/pys-2018-250201

CÁCERES, A. P.; García, A.N. 2004. "Diferenciación Interna del Espacio Geográfico de Río Gallegos: Estudio Preliminar." En: Párrafos geográficos. Revista del Instituto de Investigaciones Geográficas de la Patagonia. IGEOPAT. Año III. No 4. Universidad Nacional de la Patagonia. Comodoro Rivadavia. https://doi.org/10.18004/mem.iics/1812-9528/2015.013(01)03-004

CÁCERES, A. P., Marenco, S.; Soto, J.; Ojeda, S.; Bustos Cara, R. 1999. "Conflictos en la gestión del crecimiento urbano en sistemas municipales ejidales: Río Gallegos.” En: Revista Universitaria de Geografía. Vol. III No 1 y 2. Departamento de Geografía. Universidad Nacional del Sur. Bahía Blanca. https://doi.org/10.5962/bhl.title.140757

CÁCERES, A. P.; Norambuena, M; Frías, P.; Ampuero, C. 2017. "Nueva área rururbanaintraejidal de Río Gallegos: Barrios incorporados en el Siglo XXI". Actas Científicas CIG - $78^{\circ}$ Semana de la Geografía. GÆA.

CÁCERES, A. P., Norambuena, M.; Ampuero, C. y Triviño, G. 2013. "Consolidación de la Tercera Franja Residencial de Río Gallegos, Patagonia Argentina”. En: Contribuciones Científicas GÆA, Vol. 25. https://doi.org/10.22305/ict-unpa.v10i1.263

Expediente No 13.520-1960. Acumulado No 15.233-1961. Río Gallegos, 9 de julio de 1962.

LAURELLI, E. 2004. Nuevas Territorialidades: Desafíos para América Latina frente al siglo XXI. Ediciones Al Margen. La Plata.

Municipalidad de Río Gallegos. Ordenanza No $3259 / 98$ y modificatorias 3864/00; 3964/00; $4003 / 00 ; 4539 / 02 ; 4566 / 02 ; 4731 / 02 ; 4997 / 03 ; 5160 / 03 ; 5182 / 03 ; 5188 / 03 ; 6191 / 07$; 6406/07; 6536/08; 6576/08; 6597/08; 6705/09. Río Gallegos.

NORAMBUENA, M.; Ampuero, C.; Segovia Stanoss, M.; Cáceres, A.P. 2015. "Barrios de Inmigración Chilena en Río Gallegos. Una aproximación a la percepción de los habitantes de la ciudad"." Informes Científicos - Técnicos UNPA, 7.1. Río Gallegos. https://doi.org/10.22305/ict-unpa.v7i1.124 
NCGIA. 1990. "National Center for Geographic Information Analysis Core Curriculum en GIS” GOODCHILD, M.F.; KEMP, K. K. Centro Nacional de Información y Análisis Geográfico, Universidad de California, Santa Bárbara CA.

Portal oficial de Instituto Nacional de Estadística y Censos. https://www.indec.gob.ar

Real Academia Española RAE. 2018. Diccionario enciclopédico de la lengua española. http://www.rae.es/. https://doi.org/10.5944/epos.17.2001.10203

SPATARO, M. G. 2008. "La noción de territorialidad en la práctica profesional del Trabajo Social.” Periódico de Trabajo Social y Ciencias Sociales. Edición digital. Revista margen. Consultado en: https://www.margen.org/suscri/margen51/spataro.html. https://doi.org/10.5944/comunitania.12.3

TRIVIÑO, G. 2011. Análisis y caracterización sociodemográfica de asentamientos en la Segunda Franja Residencial de la ciudad de Río Gallegos. El caso particular del asentamiento "Madre a la lucha". Río Gallegos. Informes Científicos - Técnicos UNPA-32-2011. Río Gallegos. https://doi.org/10.22305/ict-unpa.v3i3.60

ZOIDO, F. 2000. "Diccionario de Geografía Urbana, Urbanismo y Ordenación del Territorio". Capítulos 17 y 20. Grupo ADUAR. Edición ilustrada. Barcelona, España. 